CASE REPORT

\author{
W.J. Moon \\ H.J. Kim \\ H.G. Roh \\ J. Oh \\ S.H. Han
}

\section{Diffusion Tensor Imaging and Fiber Tractography in Parry-Romberg Syndrome}

SUMMARY: We report a case of Parry-Romberg syndrome in a 32-year-old woman presenting with intermittent headache and mild sensory disturbance. MR imaging revealed minimal asymmetric atrophy of the right hemisphere with a few nonspecific white matter hyperintensities. Diffusion tensor imaging and fiber tractography, however, demonstrated clear fiber derangement, especially in the sensory tract of the right cerebral white matter.
$\mathbf{P}$ arry-Romberg syndrome (PRS) is an acquired progressive facial hemiatrophy of the skin and subcutaneous tissue, and sometimes underlying bony structure, with an occasional involvement of the nervous system ${ }^{1-5}$ The atrophic process commonly appears during the first and second decades of life and affects mainly the face below the forehead. ${ }^{6}$ The most common neurologic manifestation is epilepsy; however, there are many other neurologic features, such as migraine, facial pain, and structural brain lesions. ${ }^{1-8}$

We report a case of a patient with PRS presenting with sensory disturbance and subtle white matter changes. We confirmed white matter involvement of PRS by the use of diffusion tensor imaging (DTI) and fiber tractography with 3T MR imaging.

\section{Case Report}

A 32-year-old woman presented with intermittent headache, progressive left hemihypoesthesia (lower extremity), and right hemifacial paresthesia for 2 years. The patient recalled that the progressive facial asymmetry started 20 years previously. A physical examination revealed skull depression under the thin skin in the left frontal and right parieto-occipital region, bilateral facial hemiatrophy with alopecia, and bilateral enophthalmos, being more prominent on the left side. On a neurologic examination, the patient showed hyperactive deep tendon reflexes on both knee jerks with a left extensor plantar response, along with a peripheral facial palsy on the right side. Laboratory examinations such as blood counts, renal and hepatic function tests, hypercoagulability screening, and autoantibodies revealed normal findings.

Conventional MR images as well as DTIs were obtained by using a 3T MR imaging scanner (Signa HDx; GE Healthcare, Milwaukee, Wis). T2-weighted images and fluid-attenuated inversion recovery (FLAIR) images showed a few tiny hyperintensities in the right frontoparietal periventricular white matter, right globus pallidus, left lateral thalamus, and right upper pons (Fig 1A, -B). Mild asymmetric atrophy of the right midbrain was noted. A $3 \mathrm{D}$ volume rendering image from the T1-weighted images depicted bilateral asymmetric scalp and calvarial atrophy (Fig $1 C$ ).

DTI was performed by using a Stejskal-Tanner sequence with sin-

Received November 28, 2007; accepted after revision December 5.

From the Departments of Radiology (W.J.M., H.G.R.) and Neurology (H.J.K., J.O., S.H.H.), Konkuk University School of Medicine, Seoul, Republic of Korea.

This work was supported by the Second-Phase of BK (Brain Korea) 21 Project in 2007.

Please address correspondence to Seoul-Heui Han, MD, Department of Neurology, Konkuk University Hospital, 4-12, Hwayang-dong, Gwangjin-gu, 143-729 Seoul, Republic of Korea; e-mail:alzdoc@kuh.ac.kr

DOI 10.3174/ajnr.A0967 gle-shot spin-echo-type echo-planar imaging (TR/TE, 10,000/82 ms; matrix size, $128 \times 128$; FOV, $240 \mathrm{~mm}$; 66 axial sections; $2.4-\mathrm{mm}$ section thickness). We obtained diffusion-weighted images along 25 different directions with a b-value of $1000 \mathrm{~s} / \mathrm{mm}^{2}$, as well as an image without diffusion weighting $\left(b=0 \mathrm{~s} / \mathrm{mm}^{2}\right)$. Brain fiber tracking was performed by using software on a workstation (FuncTool; GE Healthcare). The sensory and pyramidal motor tracts were observed by the method introduced by Yamada et al. ${ }^{9}$ Fiber tractography demonstrated a markedly decreased fiber tract volume of the sensory and motor pyramidal tract on the right side. Sensory tract derangement was more prominent as compared with motor tract involvement (Fig 2).

\section{Discussion}

Up to $52 \%$ of patients with PRS have associated neuropsychiatric abnormalities such as migraine or facial pain, vision problems, depression and anxiety, and epilepsy. Among the described cases, epilepsy is the most common problem requiring patients to seek medical attention. ${ }^{1-3,6}$

In our patient, scalp and facial atrophy was bilateral but more prominent on the left frontal scalp, right parieto-occipital scalp, and left orbit. Right hemifacial paresthesia can be explained by right periorbital facial atrophy and subsequent trigeminal nerve involvement. In contrast, brain parenchymal involvement was more prominent on the right side. The right brain involvement explains clearly the left hypoesthesia of the extremities by cross-innervation of the sensory tract. In previous reports, abnormal brain lesions have been shown to be ipsilateral to the side of the face with PRS involvement. ${ }^{2-4,6,8}$

Bilaterality of PRS has been reported in approximately $2 \%$ $7.4 \%$ of cases. ${ }^{6}$ Bilateral involvement of facial atrophy and the almost unilateral involvement of the brain abnormality in our patient suggest that PRS might progress sequentially bilaterally for a long time. Bilateral involvement of facial and scalp atrophy can be visualized best by the use of $3 \mathrm{D}$ volume rendering images.

White matter and deep gray matter abnormalities on T2weighted and FLAIR images were subtle in our patient. However, fiber tractography visualized clearly asymmetric involvement of the fiber tracts and larger involvement of the fiber tracts than expected from the conventional MR images. This finding implies that axonal injury in our patient was so profound that the derangement of the fiber tract could occur. Conversely, previous case reports have demonstrated relatively preserved fiber tracts despite widespread white matter abnormalities. ${ }^{2}$ Although we could not find any cortical and subcortical abnormalities on high-resolution thin-section T1and T2-weighted images, a microscopic abnormality within the cortex might induce progressive axonal degeneration. 

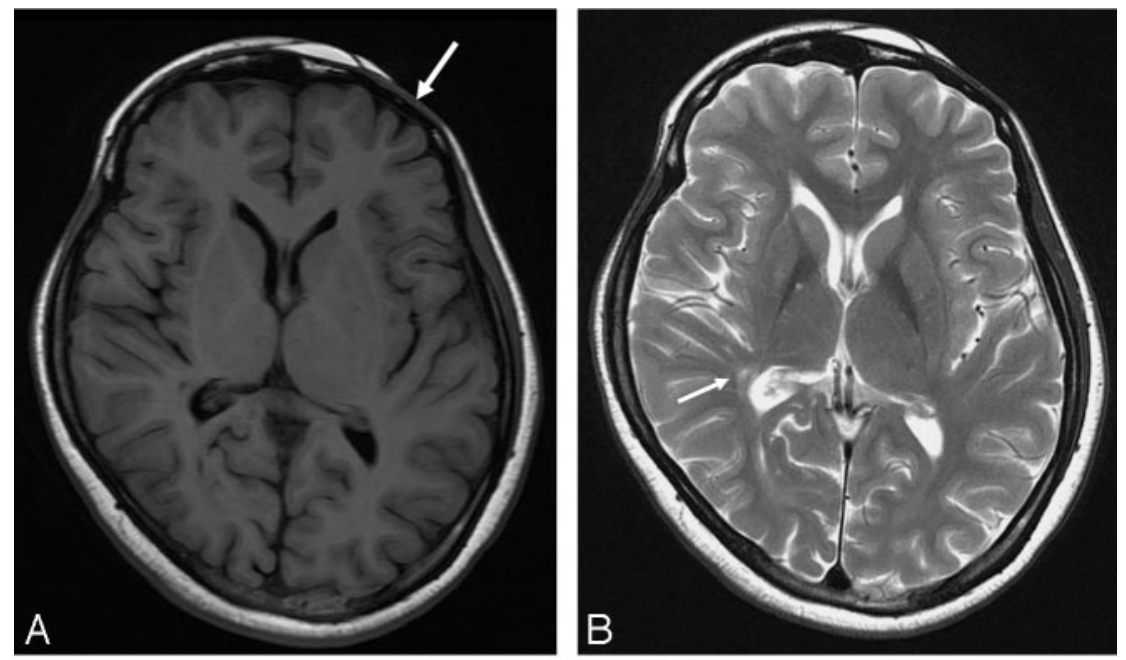

Fig 1. $A$, The T1-weighted image (at the level of basa ganglia) shows no abnormal lesion in the brain. However, atrophy of the left frontal skin and calvaria is noted (arrow). $B$, The T2-weighted image shows a few hyperintense lesions in the right basal ganglia and right posterior periventricular white matter (arrow). The midbrain appears with mild atrophy on the right side (not shown). C and D, A 3D volume rendering image shows focal skin and calvarial atrophy (arrows) over the left frontoparietal and the right parietooccipital regions.
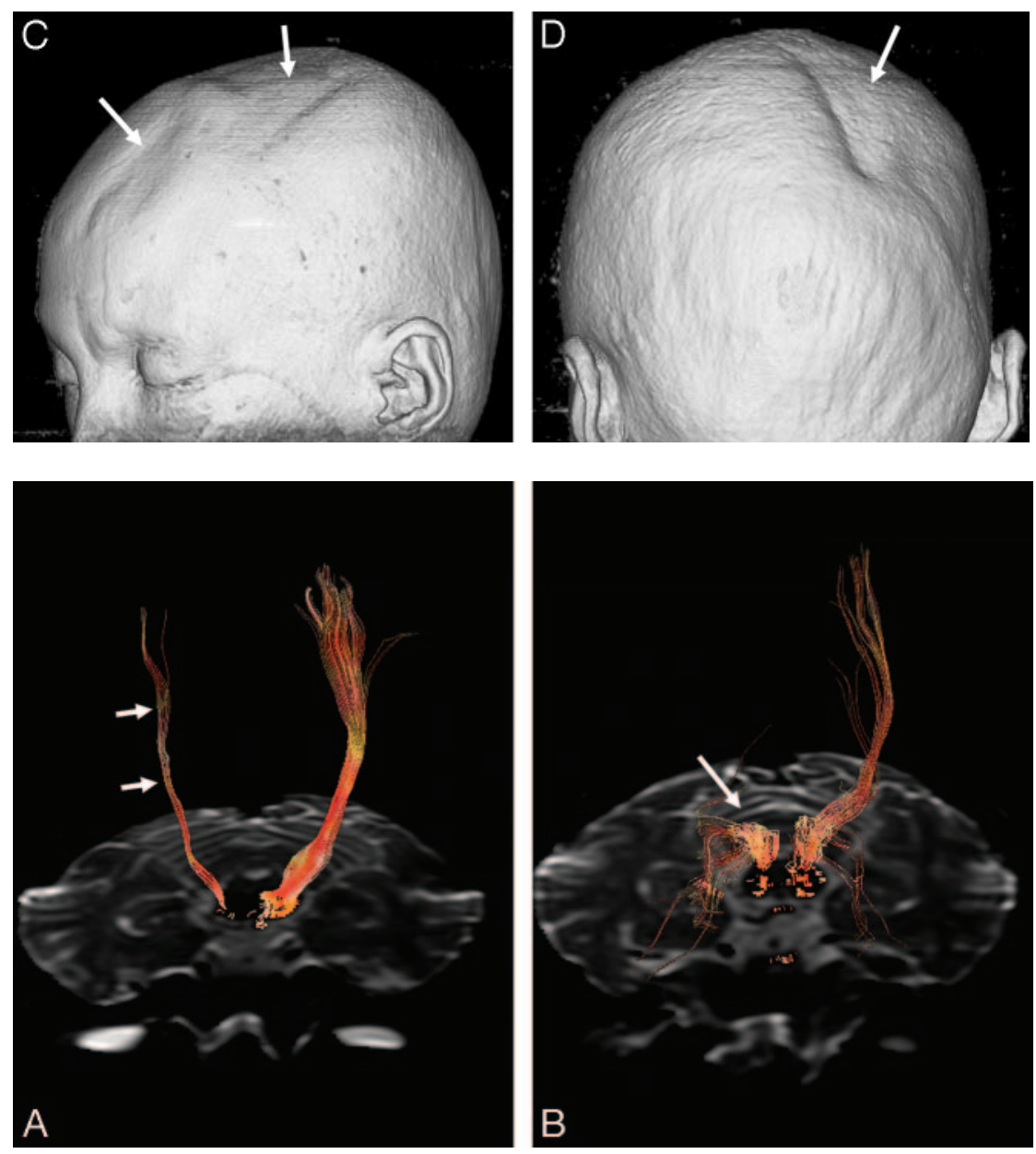

Fig 2. 3D fiber tractography of the pyramidal tracts $(A)$ and sensory tract through the dorsal pons and thalamus $(B)$. $A$, The pyramidal tracts are clearly depicted on the left hemisphere but are sparse on the right hemisphere (arrows). $B$, The sensory tract on the right side is prominently involved (arrow).

\section{References}

1. Pichiecchio A, Uggetti C, Grazia Egitto M, et al. Parry-Romberg syndrome with migraine and intracranial aneurysm. Neurology 2002;59:606-08, discussion 481

2. Okumura A, Ikuta T, Tsuji T, et al. Parry-Romberg syndrome with a clinically silent white matter lesion. AJNR Am J Neuroradiol 2006;27:1729-31

3. Paprocka J, Jamroz E, Adamek D, et al. Difficulties in differentiation of Parry-Romberg syndrome, unilateral facial sclerodermia, and Rasmussen syndrome. Childs Nerv Syst 2006;22:409-15. Epub 2005 Oct 25

The pathophysiology of PRS remains uncertain. No genetic association has been found so far. A neuroinflammatory process has been suggested as a possible pathogenic mechanism, though no associated serum autoantibodies have been found. ${ }^{1}$ The disproportionately large involvement of the fiber tracts as compared with the white matter lesion on conventional MR images in our patient suggests a widespread underlying neurodegenerative process.

In conclusion, DTI and fiber tractography are useful in evaluating the neurologic aspects of patients with PRS with the slightest involvement of the brain.
4. Moko SB, Mistry Y, Blandin de Chalain TM. Parry-Romberg syndrome: intracranial MRI appearances. J Craniomaxillofac Surg 2003;31:321-24

5. Blaszczyk M, Krolicki L, Krasu M, et al. Progressive facial hemiatrophy: central nervous system involvement and relationship with scleroderma en coup de sabre. J Rheumatol 2003;30:1997-2004

6. Tollefson MM, Witman PM. En coup de sabre morphea and Parry-Romberg syndrome: a retrospective review of 54 patients. J Am Acad Dermato 2007;56:257-63

7. Shah JR, Juhasz C, Kupsky WJ, et al. Rasmussen encephalitis associated with Parry-Romberg syndrome. Neurology 2003;61:395-97

8. Sathornsumetee S, Schanberg L, Rabinovich E, et al. Parry-Romberg syndrome with fatal brain stem involvement. J Pediatr 2005;146:429-31

9. Yamada K, Kizu O, Mori S, et al. Brain fiber tracking with clinically feasible diffusion-tensor MR imaging: initial experience. Radiology 2003;227:295-301 